\title{
Thermodynamical Approach for Choosing the Carrier System for Tetraantraquinoporphyrazines
}

\author{
Natalia Lebedeva ${ }^{1}$, Elena Malkova ${ }^{1}$, Yury Gubarev ${ }^{1}$, Anatoly V'yugin ${ }^{1}$, Albert Borisov ${ }^{2}$ \\ ${ }^{1}$ G.A. Krestov Institute of Solution Chemistry of the Russian Academy of Sciences, Ivanovo, Russia \\ ${ }^{2}$ Ivanovo State University of Chemistry and Technology, Ivanovo, Russia \\ Email: nsl@isc-ras.ru, ttoc@isuct.ru
}

Received September 30, 2013; revised October 29, 2013; accepted November 15, 2013

Copyright (C) 2013 Natalia Lebedeva et al. This is an open access article distributed under the Creative Commons Attribution License, which permits unrestricted use, distribution, and reproduction in any medium, provided the original work is properly cited.

\begin{abstract}
On the example of tetraantraquinoporphyrazines - potential photosensitizers for photodynamic therapy, thermodynamic approach to the choice of exogenous transport system was demonstrated. By means of isothermal titration calorimetry and electron absorption spectroscopy, the state of TAP in aqueous solutions was studied. The possibility of aggregation equilibrium displacement due to the pyridine adding was evaluated.
\end{abstract}

Keywords: Tetraantraquinoporphyrazines; Macroheterocycles; Dimerization; Complexation; Self-Aggregation; Transport Systems

\section{Introduction}

Metal complexes of tetraantraquinoporphyrazines (TAP) are perspective substances for practical use. High thermodynamic stability, kinetic stability and cromophore properties are provided by multicircuit conjugate aromatic system [1]. Presence of peripheral substitutes provides water solubility and allow using TAP as catalysts, photo- and chemosensors and photosensitizers. All practically useful properties of TAP depend from tendency of TAP to aggregate in solutions [2]. Self-aggregation causes tenfold decrease of biochemical, photo- and catalytic activity of macroheterocycles. That's why it is necessary to establish the influence of peripheral substitute's nature to TAP's aggregation tendency [3]. Investigations devoted to revelation of regulation mechanism for TAP state in solutions are very actual. For example, it can be the complex formation with neutral molecular ligands. Tetraantraquinoporphyrazines deserve attention as potential photosensitizers because of the presence of two types of macrocyclic systems (anthraquinon and porphyrine) providing expansion of photoactivation range from UV to long-wave region of spectrum. From the other side, the widening of aromatic system from porphyrazines to tetraantraquinoporphyrazines causes intensification of hydrophobic behavior of macroheterocycles and increases the tendency of macrocycles to self-aggregation [4]. Transport systems are necessary for using TAP as photosensitizers for photodynamic therapy of cancer. Synthetic and natural polymers can be used as carrier agents [5]. That's why information about complex formation between TAP metal complexes and electron-donor ligands is necessary for the design of transport system.

The aim of this work is to determine the thermodynamic characteristics of TAP dimers dissociation induced by molecular complex formation of TAP with electrondonor ligands.

\section{Experimental}

Sodium salts of tetraantraquinoporphyrazines (Figure 1) were synthesizes and purified according to standard procedures [6].

Crystalline samples were dried over vacuum at 343 $353 \mathrm{~K}$ to constant mass. The grade of obtained substances was examined by means of elemental analysis and electron absorption spectroscopy. Experimental data showed that grade of substances is $99.95 \%$. Very-highpurity pyridine was kept above $\mathrm{NaOH}$ during 1 - 2 days with following fractional distillation. Solutions were prepared using double distillated deionized water.

In order to evaluate thermodynamic parameters of complex formation we used isothermal titration calorimetry. Calorimetric measurements were performed with a differential automatic titration calorimeter [7]. Experimental data treatment and thermodynamic parame- 


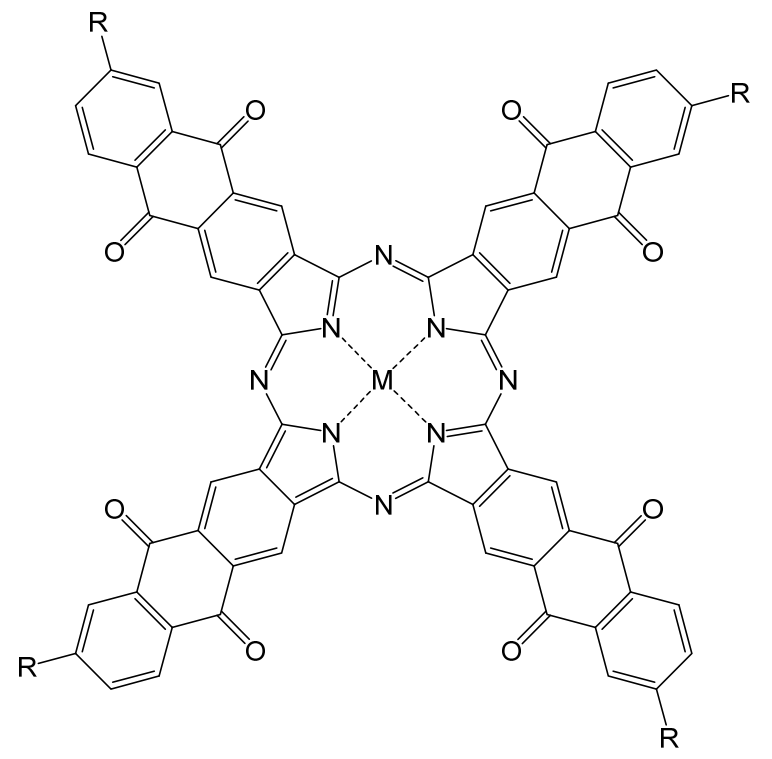

Figure 1. Molecule of tetraantraquinoporphyrazine ( $M=$ AlOH, Co; $\left.\mathrm{R}=\mathrm{COONa}, \mathrm{SO}_{3} \mathrm{Na}\right)$.

ters calculation techniques are minutely described in [810]. We took into account the following equilibriums for titration pyridine solution into solution of TAP:

$$
\begin{gathered}
(\mathrm{TAP})_{2} \stackrel{K_{1}}{\longleftrightarrow} \mathrm{TAP}+\mathrm{TAP}, \\
\mathrm{TAP}+L \stackrel{K_{2}}{\longleftrightarrow} \mathrm{TAP} \cdot L,
\end{gathered}
$$

Equilibrium constants of interdependent the first $\left(K_{1}\right)$ and the second $\left(K_{2}\right)$ processes are expressed the following way:

$$
\begin{gathered}
K_{1}=\frac{[\mathrm{TAP}]_{n}^{2}}{\left[(\mathrm{TAP})_{2}\right]_{n}} \\
=\frac{[\mathrm{TAP}]_{n}^{2}}{\left(C_{(\mathrm{TAP})_{2}}^{0}\right)-\frac{1}{2}[\mathrm{TAP}]_{n}-\frac{1}{2}[\mathrm{TAP} \cdot L]_{n}} \\
K_{2}=\frac{[\mathrm{TAP} \cdot L]_{n}}{[\mathrm{TAP}]_{n}[L]_{n}}=\frac{[\mathrm{TAP} \cdot L]_{n}}{[\mathrm{TAP}]_{n}\left(\left(C_{L}^{0}\right)_{n}-[\mathrm{TAP} \cdot L]_{n}\right)},
\end{gathered}
$$

where $[\mathrm{TAP}]_{n},\left[(\mathrm{TAP})_{2}\right]_{n},[\mathrm{TAP} \cdot L]_{n},[L]_{n}$ are equilibrium constants of TAP, TAP in dimer, molecular complex and pyridine correspondingly; $\left(C_{(\mathrm{TAP})_{2}}^{0}\right)_{n},\left(C_{L}^{0}\right)_{n}$

are initial concentrations of TAP and pyridine correspondingly after adding of $n$-th doze of titrant, $n-$ step of titration.

The energy evolved after the $n$-th injection of titrant can be determined from:

$$
Q_{n}=\Delta H_{1} V_{n}[\mathrm{TAP}]_{n}+\Delta H_{2} V_{n}[\mathrm{TAP} \cdot L]_{n},
$$

where $V_{n}$ is the volume of reaction mass.
Values of $K_{1}, K_{2}, \Delta H_{1}, \Delta H_{2}$ were determined by alternating-variable descent method combined with leastsquares method. Optimization processes was stopped when the minimum of the function

$\sum\left(Q_{n}(\exp )-Q_{n}(c a l)\right)^{2}$ was reached, $Q_{n}(c a l)$ is calculated $Q_{n}(\exp )$ is experimentally determined values of thermal effect. The descent method is unbalanced for disordered relief having some local extremums. For global minimum founding we used the random search. For all studied systems in the field of $K_{1}, K_{2}, \Delta H_{1}, \Delta H_{2}$ having physical meaning the local minimum was found. Thereby it was confirmed that the data obtained via this method are reliable. Independence of thermodynamic parameters on TAP concentration from $10^{-7} \mathrm{M}$ to $10^{-5} \mathrm{M}$ is an evidence of existence of TAP mainly in monomers and dimers in mentioned concentration range.

Electron absorption spectra of solutions of tetraantraquinoporphyrazine and phthalocyanine at additions of BSA were registered using a spectrometer Unico 2800 (United Products and Instruments, Inc., USA) in the range of $200-800 \mathrm{~nm}$. The $10 \mathrm{~mm}$ quartz cuvettes are used.

\section{Results and Discussion}

UV-VIS spectrum of HOAITAP $(\mathrm{COONa})_{4}$ is shown on Figure 2.

Concentration dependence of TAP solution absorbance showed that Lambert law did not perform. Dimerization of tetraantraquinoporphyrazines causes the decrease of Q-band intensivity (690 nm), its widening and blue shift. Such spectral changes are typical for $\pi-\pi$-dimerization. It is obviously that high-order associates' formation in the case of hydroxyaluminium tetraantraquinoporphyrazines is impossible due to steric factor (Figure 3).

Adding of pyridine to HOAlTAP $\left(\mathrm{PhSO}_{3} \mathrm{Na}\right)_{4}$ solution results in red-shift of Q-band, its widening and increase intensity while absorbance at $640 \mathrm{~nm}$ decreased. Titration is isobestic and indicative of the shift of the dimmer-monomer equilibrium to the monomer species.

According to X-ray data $[11,12]$ the distance between $\pi$ - $\pi$-interacting molecules is no more than $3-4$ A. $\pi-\pi$ dimers of hydroxoaluminium tetraantraquinoporphyrazines can't form complexes with pyridine because of axial $\mathrm{OH}$-groups on the outer side of dimer and too small internal space. Consequently the equilibrium:

$$
(\text { HOAlTAP })_{2} \leftrightarrow 2 \text { HOAlTAP }
$$

will be shifted to the right according to Le Chatelier principle due to the complex formation process:

$$
\text { HOAlTAP }+ \text { Py } \leftrightarrow \text { HOAlTAP } \cdot \text { Py }
$$

Equilibrium (6) significantly depends on anionic-cationic interactions [13]. That's why all calorimetric and 


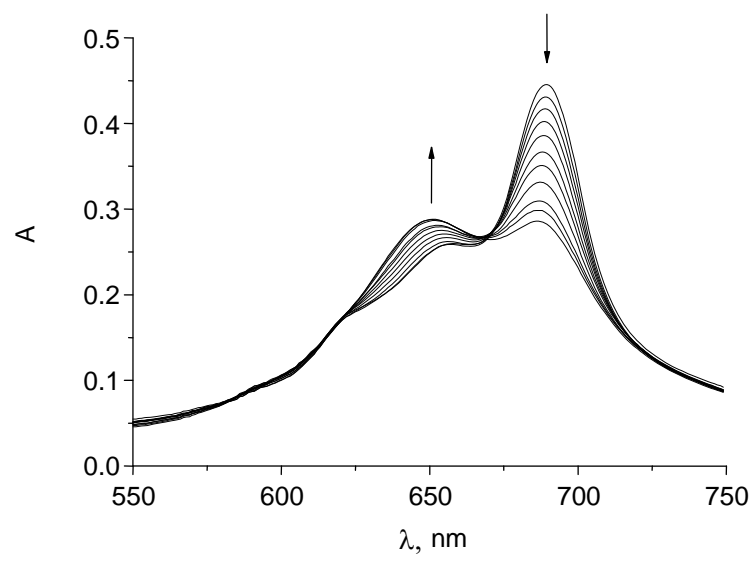

Figure 2. Electron absorption spectra of HOATTAP $(\mathrm{COONa})_{4}$ in solution (0.05 $\mathrm{M} \mathrm{NaCl}$ ) during pyridine addition (0 - 0.09 M).

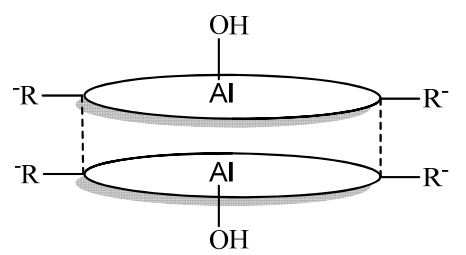

Figure 3. Layout view of TAP dimers in aqueous solutions.

spectral studies were carried out in aqueous solutions using $\mathrm{NaCl}$ as base electrolyte $(0.05 \mathrm{M})$. Use of $\mathrm{NaCl}$ as base electrolyte is necessary to eliminate salt effect. As at high total ion concentrations changes of activity coefficients of TAP vs the concentrations are not very strong, an addition of the excess of the salt results in constant activity coefficients [14]. Complex formation of TAP with pyridine causes decrease of thermodynamic stability of dimers.

Thermodynamic parameters of dimer dissociation of hydroxoaluminium tetraantraquinoporphyrazines depend on peripheral substitute's nature (Table 1).

In water-pyridine solution dimers of HOAlTAP $\left(\mathrm{SO}_{3} \mathrm{Na}\right)_{4}$ are more stable than dimers of HOAlTAP $(\mathrm{COONa})_{4}$. It can be explained by higher proton acceptor and nucleophilic ability of $\mathrm{COO}^{-}$group in compare with $\mathrm{SO}_{3}^{-}$. Electrostatic repulsion efficiency between similarly charged substitutes in the case of HOAlTAP $(\mathrm{COONa})_{4}$ decrease and causes rising of dimers stability.

Thermodynamic parameters of TAP complex formation with pyridine are totally indifferent to nature of peripherial substitutes. It can be explained by spatial remoteness of substitutes from reaction site. It is well known that inductive effect of heteroatom fully decay in 3 - 4 carbon bonds. From the other side the substitute can effect on reaction site by means of mesomeric effect and field effect. According to [15] $\mathrm{COO}^{-}$group possesses higher negative inductive effect than $\mathrm{SO}_{3}^{-}$group. Independence of thermodynamic parameters from nature of peripherial substitutes shows that tetraantraquinoporphyrazines are multicircuit conjugated systems such as phthalocyanines.

Obtained thermodynamic parameters interindependent properties (1) and (2) allows to calculate equilibrium concentration of dimers in solution for each step of titration. It also allows to determine quantities of pyridine required for equilibrium displacement to monomer species. All calculations were performed for the same concentrations of macrocycles $\left(1.2 \times 10^{-4} \mathrm{M}\right)$.

The relationship of TAP dimers percentage from pyridine quantities for HOAlTAP $\left(\mathrm{SO}_{3} \mathrm{Na}\right)_{4}$ is close to exponential (Figure 4).

Adding of more than 150-fold molar excess of pyridine into TAP solution insignificantly reflected to TAP dimers percentage. It is fundamentally important because tetraantraquinoporphyrazines are insoluble in pure pyridine and existence of high excess of pyridine in solution is undesirable.

\section{Conclusion}

The dependence between TAP dimer percentage and molar ratio Py/TAP (Figure 4) shows that pyridine is ineffective as complexing reagent for HOAITAP $(\mathrm{COONa})_{4}$. In the case of HOAITAP $(\mathrm{COONa})_{4}$, introduction of aromatic amine into carrier system is unreasonable. On the contrary, in the case of HOAlTAP $\left(\mathrm{SO}_{3} \mathrm{Na}\right)_{4}$, pyridine is effective as complexing reagent and the system contains

Table 1. Thermodynamic parameters of equilibriums (6) and (7) in aqueous solution (0.05 M NaCl) at 298.15 K.

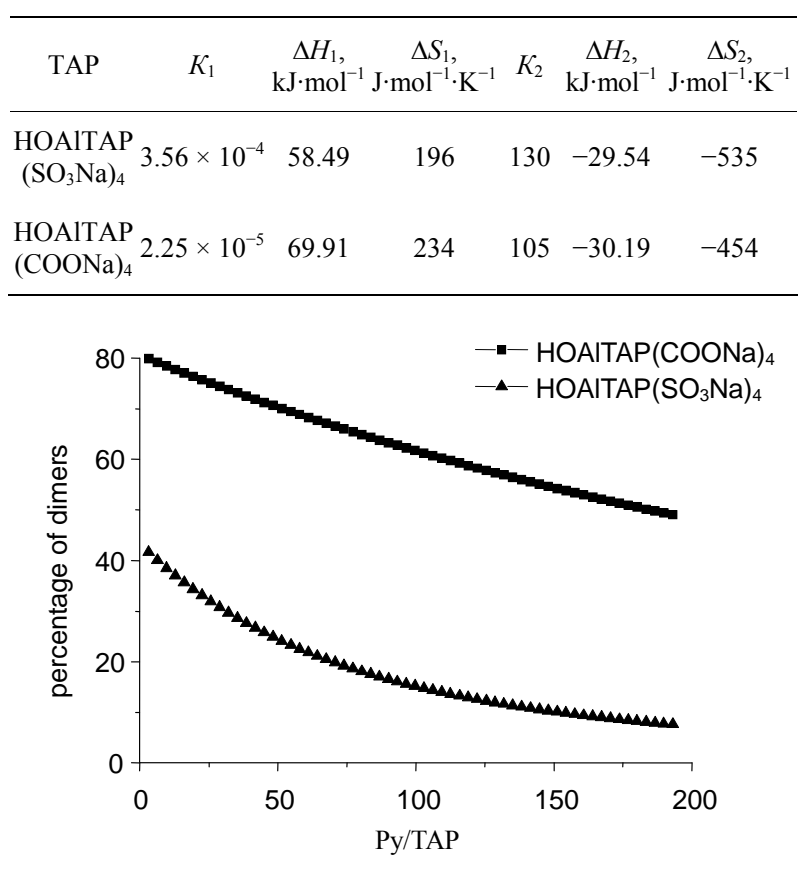

Figure 4. The dependence of TAP dimers percentage from quantities of added pyridine (molar ratio TAP/Py). 
pyridine $\mathrm{N}$-atom which can be used as the carrier system.

\section{Acknowledgements}

This work was supported by the fellowship of Russian Foundation for Basic Research 12-03-97524-r_centr_a.

\section{REFERENCES}

[1] M. T. Nunes, F. S. Sguilla and A. C. Tedesco, "Photophysical Studies of Zinc Phthalocyanine and Chloroaluminum Phthalocyanine Incorporated into Liposomes in the Presence of Additives," Brazilian Journal of Medical and Biological Research, Vol. 37, No. 2, 2004, pp. 273284. http://dx.doi.org/10.1590/S0100-879X2004000200016

[2] P. Zimchik, V. Novakova, M. Miletin and K. Kopetcky, "Azaphthalocyanines Containing Pyrazine Rings with Focus on the Alkylheteroatom, Aryl and Heteroaryl Substitution and Properties Important in Photodynamic Therapy," Macroheterocycles, Vol. 1, No. 1, 2008, pp. 21-29.

[3] M. E. Rodriguez, F. Morán, A. Bonansea, M. Monetti and D. A. Fernández, "A Comparative Study of the Photophysical and Phototoxic Properties of Octakis (Decyloxy) Phthalocyaninato Zinc(II), Incorporated in a Hydrophilic Polymer, in Liposomes and in Non-Ionic Micelles," Photochemical \& Photobiological Sciences, Vol. 2, No. 10, 2003, pp. 988-994. http://dx.doi.org/10.1039/b303428n

[4] I. L. Kogan and K. Yakushi, "New Conducting Composite Material Based on a Platinum Phthalocyanine Charge Transfer Salt and Poly(bisphenol A Carbonate): Electrochemical, Optical, Magnetic and Structural Properties," Journal of Materials Chemistry, Vol. 7, 1997, pp. 22312234. http://dx.doi.org/10.1039/a702670f

[5] G. Valduga, A. Pifferi, P. Taroni, G. Valentini, E. Reddi, R. Cubeddu and G Jori, "Steady-State and Time-Resolved Spectroscopic Studies on Low-Density Lipoprotein-Bound Zn(II)-Phthalocyanine," Journal of Photochemistry and Photobiology, Vol. 49, No. 2-3, 1999, pp. 198-203. http://dx.doi.org/10.1016/S1011-1344(99)00059-7

[6] A. Borisov, V. Maizlish and G. Shaposhnikov, "Tetraanthraquinonoporphyrazines: II. Synthesis and Properties of Metal Complexes of Substituted Tetraanthraquinonoporphyrazines," Russian Journal of General Chemistry, Vol. 75, No. 10, 2005, pp. 1579-1583. http://dx.doi.org/10.1007/s11176-005-0470-0
[7] N. Sh. Lebedeva, K. V. Michailovsky and A. I. V'yugin, "A Differential Automatic Titration Calorimeter," Russian Journal of Physical Chemistry A, Vol. 75, No. 6, 2001, pp. 1031-1033.

[8] N. Sh. Lebedeva, N. A. Pavlycheva and O. V. Petrova, "Determination of the Dimerization Constants of Water Soluble Metallophthalocyanines by Calorimetric Titration Using Electron-Donating Ligands," Mendeleev Communications, Vol. 3, No. 5, 2003, pp. 237-238. http://dx.doi.org/10.1070/MC2003v013n05ABEH001767

[9] N. Sh. Lebedeva, N. A. Pavlycheva, A. I. Vyugin, V. E. Maizlish and G. P. Shaposhnikov, "Complex-Formation Thermodynamics of Water-Soluble Metal Phthalocyanines with Pyridine," Russian Journal of Inorganic Chemistry, Vol. 49, No. 2, 2004, pp. 289-293.

[10] N. Sh. Lebedeva, N. A. Pavlycheva, O. V. Petrova and E. V. Parfenyuk, "Effect of Molecular Complex Formation of Metallophthalocyanines with Pyridine on Their Aggregation," Journal of Porphyrins and Phthalocyanines, Vol. 9, No. 4, 2005, pp. 240-247.

http://dx.doi.org/10.1142/S1088424605000307

[11] R. Caminiti, A. Capobianchi, P. Marovino, A. M. Paoletti, G. Padeletti, G. Pennesi and G. Rossi, "Structural and Morphological Characterisation of Ruthenium Phthalocyanine Films by Energy Dispersive X-Ray Diffraction and Atomic Force Microscopy," Thin Solid Films, Vol. 382, No. 1, 2001, pp. 74-80. http://dx.doi.org/10.1016/S0040-6090(00)01701-6

[12] J. Silva, J. Brito, S. Tanimoto and N. Souza, "Morphological Structure Characterization of PAH/NiTsPc Multilayer Nanostructured Films," Materials Sciences and Applications, Vol. 2, No. 11, 2011, pp. 1661-1666. http://dx.doi.org/10.4236/msa.2011.211221

[13] N. Sh. Lebedeva, "Aggregation Properties of WaterSoluble Metal Phthalocyanines: Effect of Ionic Strength of Solution," Russian Chemical Bulletin, International Edition, Vol. 53, No. 12, 2004, pp. 2674-2683.

[14] D. D. Perrin, "Stability Constants of Metal-Ion Complexes. Part B. Organic Ligands," Pergamon Press, London, 1979.

[15] S. Lupton, "Field and Resonance Components of Substituent Effects," Journal of the American Chemical Society, Vol. 90, No. 16, 1968, pp. 4328-4337. http://dx.doi.org/10.1021/ja01018a024 